\title{
Model Based Analysis of Shimmy in a Racing Bicycle
}

\author{
Nicolò Tomiati ${ }^{1} \quad$ Gianantonio Magnani $^{1} \quad$ Bruno Scaglioni $^{1} \quad$ Gianni Ferretti $^{1}$ \\ ${ }^{1}$ Politecnico Di Milano, Dipartimento di Elettronica, Informazione e Bioingegneria DEIB \\ Via Ponzio 34/5, 20133 Milano, Italy, \\ \{gianantonio.magnani, bruno.scaglioni,gianni.ferretti\}@polimi.it, \\ nicola.tomiati@mail.polimi.it
}

\begin{abstract}
In this paper we are presenting a model of a racing bicycle, developed in Modelica language within the Dymola environment. The main purpose is to investigate the dynamic response of the bicycle and its modes of vibration, referring in particular to shimmy. This phenomenon occurs at high speeds and consists of sudden oscillations of the front assembly around the steering axis. Lateral accelerations on the horizontal tube of the frame can reach 5-10 g with a frequency that varies between $5-10 \mathrm{~Hz}$. Even if it is quite uncommon, shimmy is a topic of great relevance, because it may be extremely dangerous for the rider. Thanks to this model, we can show that the main elements which contribute to the rise of the oscillations are the lateral compliance of the frame and the tyres' deformation.

Keywords: bicycle, shimmy, flexible multibody systems
\end{abstract}

\section{Introduction}

This paper will present a multibody model of a racing bicycle developed in Modelica, within the Dymola environment. The main purpose of this work is to investigate in depth the dynamic response of the bicycle and its modes, referring in particular to shimmy.

Any two-wheeled vehicle is subject, during its movement, to three modes of vibration: capsize, weave and wobble. The first two are always present; the third one occurs occasionally.

If the capsize mode is unstable, the bicycle follows a spiral path with increasing values of the roll angle that leads it to a lateral fall.

The weave mode consists, instead, in an oscillatory motion of the rear frame about the yaw axis together with oscillations about the roll axis. In this case, the frequency is of 1-2 Hz.

Finally, the wobble mode (which is often referred to as shimmy) is an oscillatory motion of the front assembly with respect to the steering axis. When it occurs, lateral accelerations on the horizontal tube of the frame can reach 5-10 $\mathrm{g}$ with a frequency that varies between $5-10 \mathrm{~Hz}$ (Magnani, Ceriani, and Papadopoulos 2013). This phenomenon is therefore very violent, unexpected and can lead to dramatic consequences, particularly if the rider does not know it and is not able to handle it. Fortunately, it does not occur so frequently and it is difficult that it can lead to a fall, although this is the sensation perceived by the cyclist. Usually, this happens at high speed, such as the one that can be reached along a downhill road. The phenomenon is well known among cyclists and bicycle manufacturers. It is a topic of great relevance because it is not still clear what are the main causes that lead to these vibrations.

Thanks to experimental activities (Magnani, Ceriani, and Papadopoulos 2013) and by using numerical models (Plöchl et al. 2012; Klinger et al. 2014; Limebeer and Sharp 2006), the lateral compliance of the frame and the tyres' deformation have been found to be two essential contributors to the wobble mode. One of the goals of this article is to understand in detail what are the causes or factors that excite these vibrations, referring in particular to a racing bicycle.

The paper is organised as follows. Section 2 gives an overview of the overall bicycle model, describing all the components in detail. Section 3 explains how the elements are connected to each other and what assumptions have been made before running the simulations. In Section 4 simulation results are presented. Two different versions of the model will be analysed. At the end, in Section 5 the conclusions and some possible practical advice that may be helpful to the rider to damp out the shimmy oscillations are discussed.

\section{Bicycle Model}

The multibody model presented in this work is based (for some components) on the Modelica MotorcycleDynamics package, which is described in detail in (Donida, Ferretti, Savaresi, Schiavo, et al. 2006; Donida, Ferretti, Savaresi, and Tanelli 2008). This library, in turn, was developed by VehicleDynamics, which shares basically the same structure (Andreasson 2003).

The following step is to run simulations to study its dynamic behaviour. Our attention has been focused on a racing bicycle, which is described in more detail in (Klinger et al. 2014). Whenever possible, therefore, data reported in that article has been used in order to make the model as compliant as possible to the real behaviour.

The main components of the model are:

- the rear frame; 


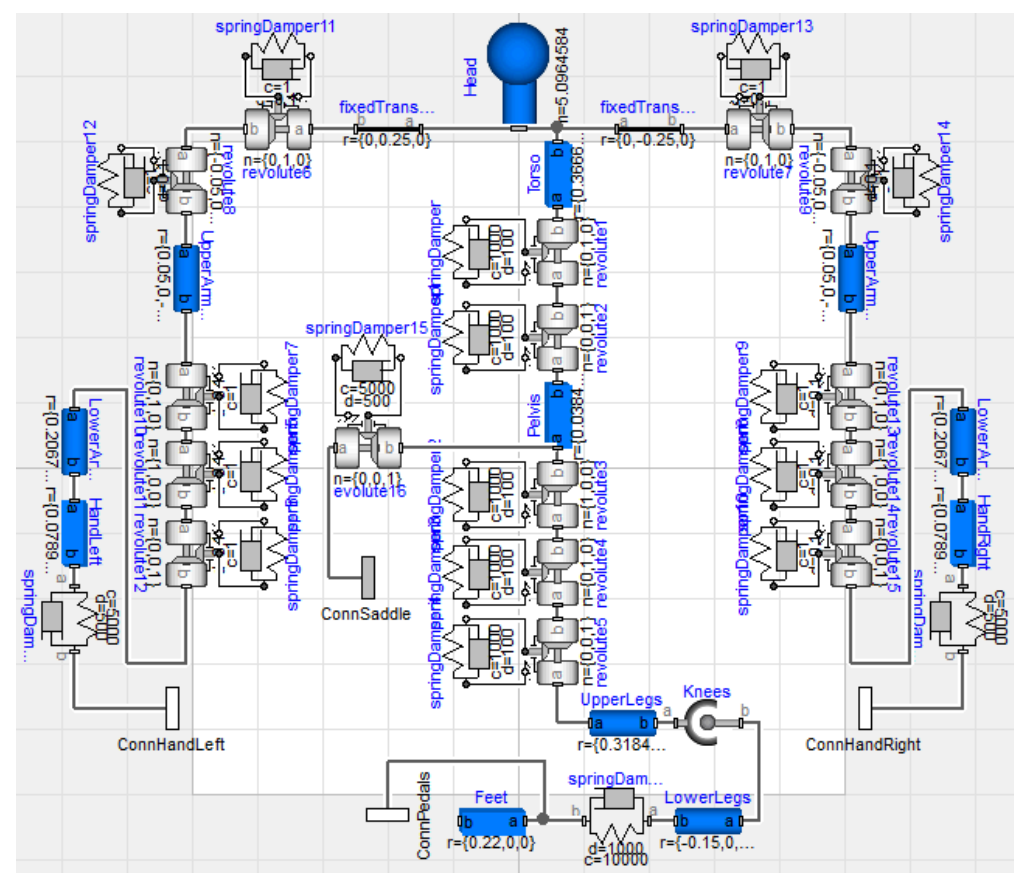

Figure 1. Rider block diagram in Dymola with the four interfaces to connect it to the other components. Four spring-damper elements have been introduced to model the compliance of the constraints between rider's hands-handlebar, feet-pedals and pelvissaddle.

- the front assembly, which includes handlebar, stem and fork;

- the cyclist;

- the front and rear wheels;

- the road.

\subsection{Rear Frame}

The first component is modelled by a BodyShape element, i.e. a single rigid body characterised by centre frame, mass and inertia tensor. In order to associate to this body the true shape of the frame, we have used a CAD model. Secondly, we have added the saddle, which is connected to the rear frame with a Revolute joint. This type of connection allows the rotations around an axis passing through the saddle tube. In this way, it is possible to consider the compliance of the constraint between the saddle and the frame.

The rear frame model presents four interfaces that allow connecting this component with the rider (including the saddle and pedals), with the front assembly (through the steering axis) and with the rear wheel (at the hub).

\subsection{Front Assembly}

The front assembly has also been modelled as a rigid body with its inertia tensor and whose mass is concentrated in a single point. It consists of the fork, whose true shape has been defined in a CAD model, the stem and the handlebar. Four interfaces characterise this component; in fact, the front assembly can be connected with the rear frame, with the front wheel at the hub, and with the cyclist at the two contact points on the handlebar.

\subsection{Cyclist}

The third component of the model is the rider. It has been modelled as a multibody system obtained by the connection of solid geometric elements having different shapes. In particular, Cylindrical elements have been used to model limbs (i.e. arms, forearms, thighs and legs) while Rectangular parallelepipeds for the torso, the pelvis, hands and feet. In regards to the head, a Body element has been chosen, which is characterised by mass and inertia tensor. It is visualised by a cylinder and by a sphere that has its centre at the centre of mass.

To model the human articulations two types of joints have been used, chosen depending on the possible relative movements between the parts connected. Spherical joints prevent all the translations but enable the rotations about three mutually orthogonal axes. On the other hand, Revolute joints prevent all the translations and the rotations about two axes. Therefore, they leave only one degree of freedom (a rotation about an axis). It is important to notice that a Spherical joint can be obtained by connecting to one another three Revolute joints, specifying for these objects three orthogonally axes of rotation (as has been done with the elbows).

To make the model more realistic, elements made up of a spring and a damper in parallel have been added: in this way it is also possible to take into account the contribution of stiffness and damping of human muscles. Figure 1 shows the rider block diagram in Dymola. Four interfaces 

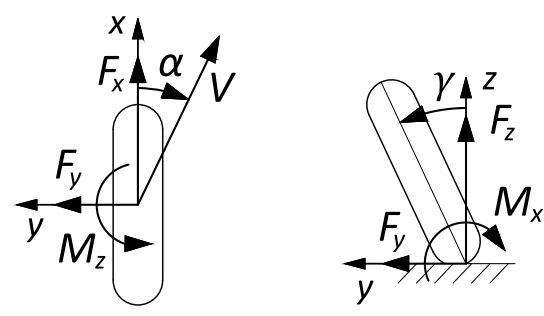

Figure 2. Tyre definitions: side-slip angle $\alpha$ is defined as the angle between the wheel centre plane and the direction of the forward velocity $V$. Camber angle $\gamma$ is defined as the angle between the wheel centre plane and the vertical axis $z$ of the road. $F_{x}$ is the longitudinal force, $F_{y}$ is the lateral force and $F_{z}$ is the normal force. $M_{x}$ is the overturning torque and $M_{z}$ is the aligning torque. Positive values are shown. The left figure is a top view while the right one is a rear view.

have been included in the model. In so doing, the rider can be connected to the front assembly and to the rear frame. Two spring-damper elements have been added to cyclist's hands and the upper part of the front assembly to model his grip on the handlebar. The same has been done for the connection between rider's feet and bicycle pedals. Lastly, a spring-damper element has also been added between the cyclist's pelvis and the saddle in order to model the compliance of the sitting position (the rider, in fact, is not rigidly attached to the saddle).

In order to verify that the behaviour of the model was compatible with a rider's real movements, different simulations have been performed (for example, by simulating a turning manoeuvre or the execution of a curved trajectory).

\subsection{Wheel Model}

Wheels are modelled as rigid bodies with their mass concentrated in the hub. Afterwards, a torus model has been used to associate the real tyre shape to the wheel. The front and rear wheels have the same dimensions (i.e. the same radius), but different mass and inertial properties.

\subsection{Tyres and Wheel-Road Interaction}

As already mentioned in the Introduction, to highlight the wobble mode it is necessary to consider tyres' deformation.

The tyre allows the contact between the rigid part of the wheel (i.e. the hub) and the road surface. At the same time, it ensures adherence to the asphalt and generates distributed forces and torques within the contact region. In the following, it will be assumed that these forces and torques are instead concentrated and applied at the single contact point that represents the interaction between wheel and road surface. In order to compute these forces, four reference frames are needed, as explained in (Donida, Ferretti, Savaresi, Schiavo, et al. 2006). Figure 2 shows

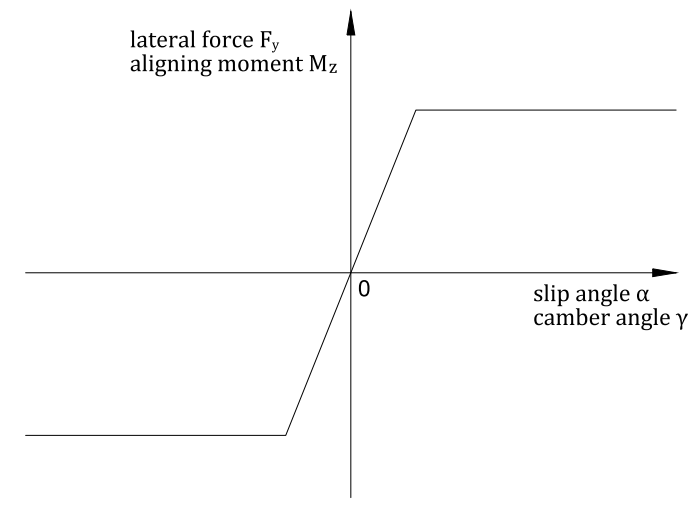

Figure 3. Qualitative trend of the lateral force $F_{y}$ and aligning torque $M_{z}$ as a function of the side-slip $\alpha$ and camber $\gamma$ angles.

the sign convention adopted in this work.

As can be seen, $\alpha$ is the side-slip angle, which is defined as the angle between the forward velocity $V$ and the wheel centre plane; $\gamma$ is instead the camber angle, defined as the angle between the vertical axis $z$ of the road and the wheel centre plane.

The following step is the determination of contact forces and torques. As stated in (Pacejka 2006), there are different relations between forces and angles. For our purposes, a linear relation has been chosen to describe tyres behaviour. Moreover, the model has taken into account the tyres' dynamic, i.e. the delay in the deformation due to the elasticity properties of the material. The tyre, in fact, does not respond immediately when it is rolled from the standstill under a slip angle. It is necessary some time before the lateral force $F_{y}$ approaches the stationary value. The same is true for the aligning torque $M_{z}$.

The longitudinal force $F_{x}$, which can represent both traction and braking forces, is defined as:

$$
F_{x}=C_{F \kappa} \kappa
$$

where $\kappa$ is the longitudinal wheel slip.

On the other hand, the lateral force $F_{y}$ is the sum of two terms:

$$
F_{y}=C_{F \alpha} \alpha^{\prime}+C_{F \gamma} \gamma^{\prime}
$$

The aligning torque $M_{z}$ also depends on both the side-slip angle $\alpha^{\prime}$ and the camber angle $\gamma^{\prime}$, according to this equation:

$$
M_{z}=-C_{M \alpha} \alpha^{\prime}+C_{M \gamma} \gamma^{\prime}
$$

The side-slip angle $\alpha^{\prime}$ in (2) and (3) differs from $\alpha$ because of the delay in the tyre response after the deformation. The same is true for the camber angle $\gamma^{\prime}$. These dynamics have been modelled by two first-order differential equations, i.e.:

$$
\begin{aligned}
& \frac{\sigma_{\alpha}}{V_{x}} \dot{\alpha}^{\prime}+\alpha^{\prime}=\alpha, \\
& \frac{\sigma_{\gamma}}{V_{x}} \dot{\gamma}^{\prime}+\gamma^{\prime}=\gamma,
\end{aligned}
$$


where $V_{x}$ is the longitudinal component of the forward velocity. The characterising parameter, called relaxation length $\sigma$, is similar to a time constant except that it has units of length rather than time. The relaxation length is a tyre characteristic that can be determined experimentally (Limebeer and Sharp 2006).

Finally, the overturning torque $M_{x}$ has been also considered, defined as:

$$
M_{x}=C_{M x} \gamma
$$

To further improve the model, two saturation limits with respect to the lateral force $F_{y}$ and the aligning torque $M_{z}$ have been introduced. This means that, at high values of side-slip and camber angles, this force and torque are constant (see Figure 3). In this way, the trend of the curve is very similar to the one that can be obtained by applying the Pacejka's magic formula described in (Pacejka 1993). The linear approximation is valid only for small values of the two angles.

The stiffness coefficients inside equations (1)-(6) depend on the vertical force $F_{z}$ transmitted on the ground at the contact point between tyre and road surface. Dymola computes its value at any given time (typically, in fact, the vertical force $F_{z}$ is not constant during the movement) and this operation allows to compute all the stiffness coefficients. When contact forces and torques are known, a balance is carried out at the hub, i.e. the point where the wheel is connected to the other components of the bicycle.

\subsection{Road}

The road surface has been modelled through the Environments package of the MotorcycleDynamics library.

This package allows the user to select the road slope (level, uphill or downhill road) and its characteristics (dry asphalt, wet and so on). To run the simulations it has been chosen to work with a dry road, having a slope such as the bicycle forward speed increases linearly from $10 \mathrm{~m} / \mathrm{s}$ to $20 \mathrm{~m} / \mathrm{s}$ in 40 seconds (see Figure 4). From the results of the experimental activity described in (Magnani, Ceriani, and Papadopoulos 2013), it is shown that shimmy appears in this speed interval. The quote $z=f(x, y)$ of the road surface is defined by the equations:

$$
z=\left\{\begin{array}{rl}
0 & \text { if } x<0 \\
-0.035 k(x) x & \text { if } x \geq 0
\end{array},\right.
$$

where $x$ is the position along the longitudinal direction, while:

$$
k(x)=\frac{\arctan \left(10 x+\frac{\pi}{2}\right)}{\pi} .
$$

Equation (8) is necessary to avoid discontinuities on the road surface, i.e. it guarantees an appropriate connection when the road slope changes.

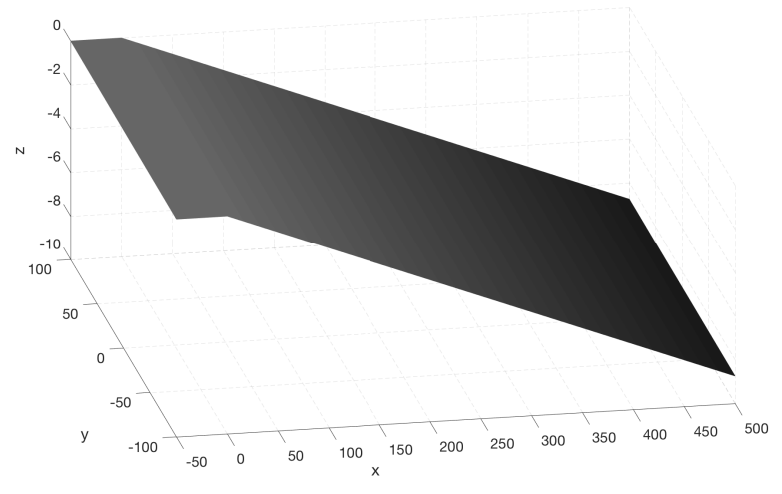

Figure 4. Road surface.

\section{Model Assembly}

Figure 5 shows the connections between the different models.

In more detail, the rider is connected to the rear frame and to the front assembly, including the saddle, the pedals and the two contact points on the handlebar. The front wheel is attached to the hub of the front assembly with a Revolute joint. This element simulates the behaviour of the ball-bearing. Similarly, the rear wheel is attached to the bicycle main frame.

Lastly, it is necessary to connect to one another the front assembly and the rear frame. Once again, a Revolute joint has been used: it introduces the rotation $\delta$ of the steering axis. As previously mentioned, there is another key element that is essential to trigger the wobble mode. This is the lateral compliance of the frame and it can be modelled by a second Revolute joint that allows the rotations of the front assembly around the $\beta$-axis (see Figure 6).

This axis is in the plane of symmetry of the vehicle and it is perpendicular to the steering axis, as suggested in (Klinger et al. 2014). The flexibility is lumped at the steering head. The user can set the values of stiffness $k_{\beta}$ and damping $c_{\beta}$ coefficients that represent the structural properties of the frame. Figure 7 shows the three-dimensional representation of the rider-bicycle model. As can be seen, the cyclist assumes the typical position for riding a racing bicycle, with his upper body in a bent-forward position and his hands firmly attached to the handlebar.

Some other simplifying assumptions are also needed. The gravity force acts on each component, and the aerodynamic drag force ${ }^{1}$ has been neglected, assuming that the contribution related to this force is balanced by the component of the weight that appears when the bicycle is moving on a downhill road. Moreover, it has been assumed that the aerodynamic force does not change the vertical forces $F_{z}$ acting on the wheels' contact points. Actually, the lift force reduces the vertical load on both front and

\footnotetext{
${ }^{1}$ The aerodynamic force can be divided into two components: drag force, which is directed along the longitudinal axis, and lift force, which is directed along the vertical axis.
} 


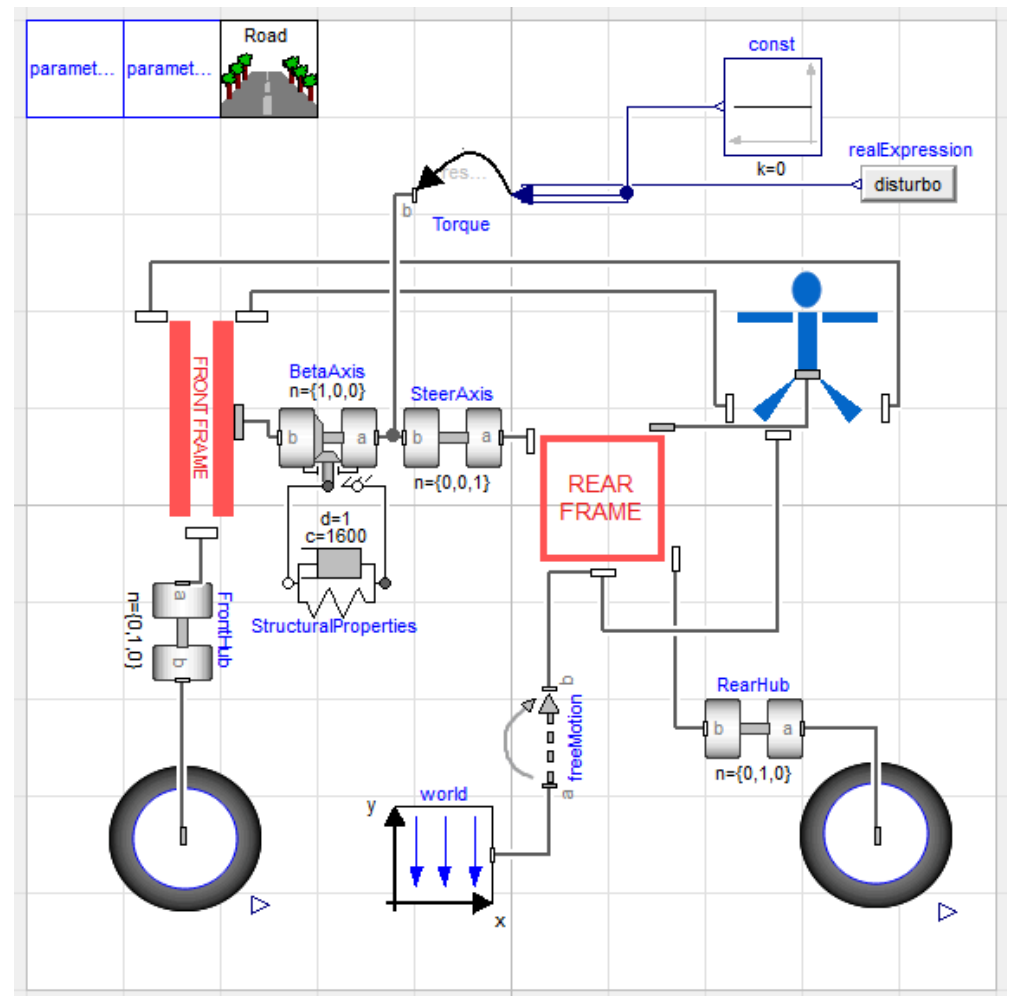

Figure 5. Model assembly that highlights the connections between components. A Revolute joint with a spring-damper element has been added to model the frame lateral compliance ( $\beta$-axis).

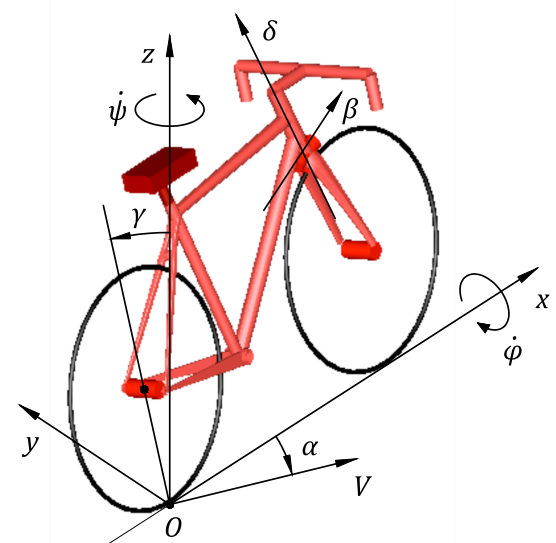

Figure 6. This figure shows the steering axis $\delta$, the axis $\beta$ that is necessary to model the frame lateral compliance, $\operatorname{roll}(\dot{\varphi})$ and yaw $(\dot{\psi})$ angular velocities, the camber angle $\gamma$ and the side-slip angle $\alpha$. Positive values are shown.

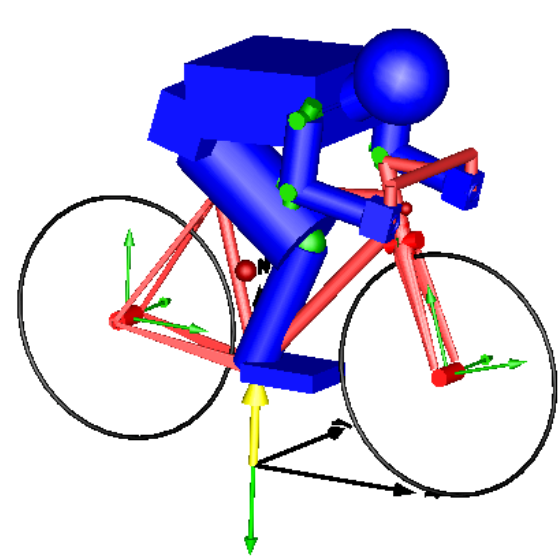

Figure 7. Three-dimensional representation of the racing bicycle model. 


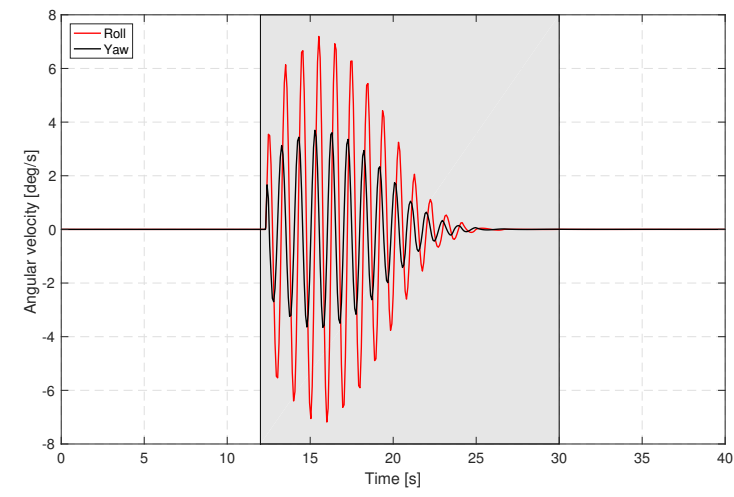

Figure 8. Roll and yaw angular velocities of the bicycle rear frame when $k_{\beta} \rightarrow \infty$ (rigid frame).

rear tyres, while the drag force increases the rear vertical load and decreases the front one.

\section{Simulation Results}

The aim of the simulations is to study the model dynamic response after the application of suitable perturbations, trying to point out the wobble mode. For this reason, an impulsive torque disturbance has been chosen. It is applied on the steering axis when the forward speed is equal to $v_{s}=13 \mathrm{~m} / \mathrm{s}$.

\subsection{Rigid Frame Model}

The first scenario considered is characterised by a rigid version of the bicycle model. It can be obtained by setting the frame stiffness coefficient $k_{\beta} \rightarrow \infty$. After the torque application, the steering axis is subject to oscillations that initially increase in amplitude and then decrease up to being completely damped. However, their frequency is approximately equal to $1 \mathrm{~Hz}$, a value much smaller than 5$10 \mathrm{~Hz}$ that characterises the wobble mode. Although other simulations have been carried out by changing the type of the perturbation and some model parameters, we have not been able to trigger the shimmy using the bicycle model with a rigid frame. Figure 8 shows, instead, the rear frame roll and yaw angular velocities.

The oscillation trend is the same that characterises the steering axis response, i.e. with oscillations that initially increase and then disappear after a few seconds. As can be noticed, the two signals have a phase difference of $90^{\circ}$ : when the roll angular velocity is zero, the yaw rate reaches its maximum (or minimum). This trend perfectly describes the weave mode. More specifically, supposing the rider to be sitting on the saddle, when a counter-clockwise torque is applied to the steering axis $\delta$, the bicycle initially rotates counter-clockwise about the yaw axis $z$ and then clockwise about the longitudinal axis $x$ (see Figure 6) ${ }^{2}$.

\footnotetext{
${ }^{2}$ This movement is consistent with the so-called countersteering: for example, to perform a right curve at high speed, what is being done is slightly push the handlebar as if you were to turn in the opposite direction (i.e. to the left). The bicycle responds by leaning correctly in
}

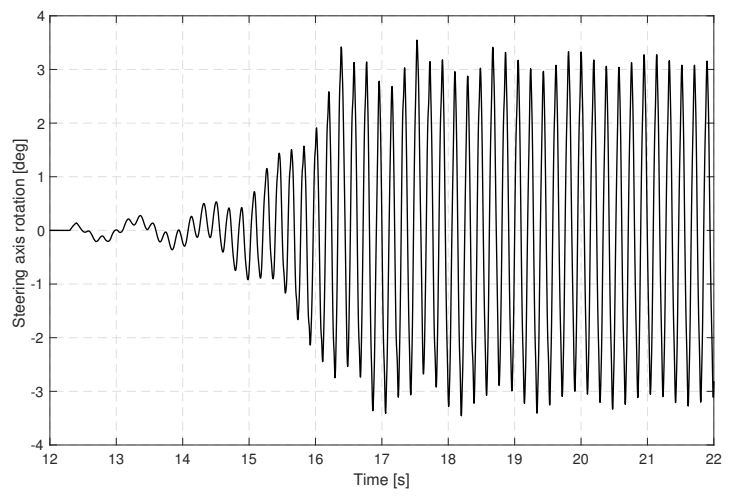

Figure 9. Zoom of the steering rotation response for the lumped flexibility frame model.

The oscillations related to the weave mode are damped because the weave eigenvalue computed on the linearized model passes through the imaginary axis, i.e. from the instability region of the complex plane (the right half-plane) to the stability area (the left half-plane). If this does not occur, the oscillations are different (not damped) and they lead to a fall of the bicycle.

\subsection{Lumped Flexibility Frame Model}

Simulations have been repeated considering the lateral compliance of the frame (hereinafter referred to as lumped flexibility frame model). A zoom of the steering axis response after the torque disturbance application is shown in Figure 9.

As can be seen, the model response to the disturbance consists of low-frequency oscillations with small amplitude (some tenths of a degree) together with highfrequency oscillations. Steering rotation reaches in a few seconds an amplitude of some degrees. Thanks to the saturation imposed to the lateral force $F_{y}$ and to the aligning torque $M_{z}$, the oscillations do not diverge but their amplitude is limited in time. The initial behaviour of the steering rotation of the lumped flexibility frame model is very similar to the one that characterises the rigid version of the bicycle. This means that the degree of freedom which represents the lateral compliance $\beta$ is, therefore, essential for the high-frequency contribution in the system response. Figure 10 shows the spectrograms related to roll and yaw angular velocities ${ }^{3}$.

As in the previous simulations, by applying the torque disturbance the weave mode is excited. Its frequency is now $f_{\text {weave }}=0,98 \mathrm{~Hz}$. This mode is also stable: after a few seconds, in fact, the oscillations disappear because they are damped. When it happens, only the high-frequency oscillations remain in the system response. They represent the wobble mode. As can be seen from Figure 10, these oscillations are characterised by a fre-

the curve direction (Åström, Klein, and Lennartsson 2005).

${ }^{3} \mathrm{~A}$ spectrogram is a visual representation of the spectrum of frequencies in a signal as it varies with time. 


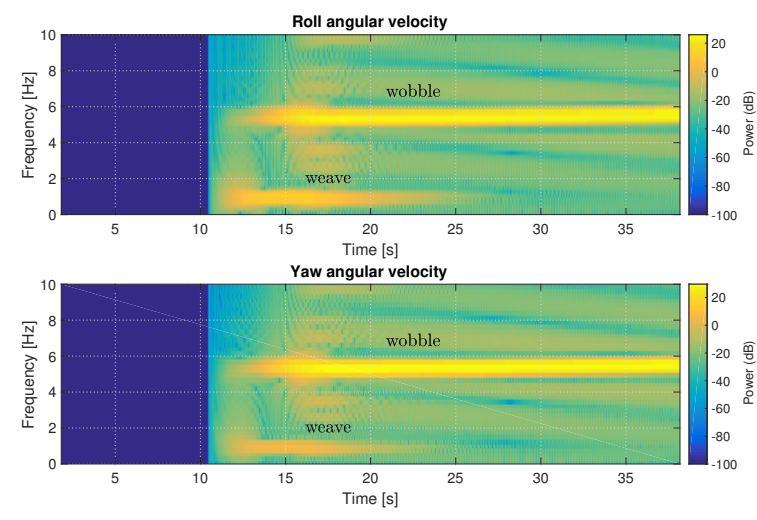

Figure 10. Spectrograms of roll and yaw angular velocities for the flexible bicycle. As can be noticed, the wobble frequency is independent with respect to the forward speed.

quency equal to $f_{\text {wobble }}=5,43 \mathrm{~Hz}$. In the experimental activity described in (Magnani, Ceriani, and Papadopoulos 2013) it is reported that the frequency of shimmy for this particular racing bicycle is $7,5 \mathrm{~Hz}$. This value is higher than the one obtained by the lumped flexibility frame model. By running other simulations, it was noted that the wobble frequency $f_{\text {wobble }}$ changes varying the value of the parameter related to the frame stiffness, i.e. $k_{\beta}$. The same result can be achieved by changing the parameters of the spring-damper combination that models the rider's hand grip on the handlebar.

In (Magnani, Ceriani, and Papadopoulos 2013) it is said that the wobble frequency seems to be independent with respect to the bicycle's forward speed: this important result has been obtained also through the Dymola model (see again Figure 10).

\section{Concluding Remarks}

This work presented the development of a racing bicycle model in Modelica language. The model has been built trying to make it as compliant as possible to the real behaviour. For this reason, attention has been focused on the rider and on the wheel-road interaction.

By running simulations with the rigid model (without the frame lateral compliance), the only vibrational mode that has been excited is the weave mode. It has been necessary to modify the model by introducing an additional degree of freedom to highlight the wobble mode. This shows that it is necessary to consider both the frame lateral compliance and the tyres' deformation (also by taking into account their dynamic behaviour) to trigger the highfrequency oscillations characterising the shimmy.

The wobble mode appears when the forces and torques that arise at the contact point of the front wheel are larger than the value needed to guarantee the longitudinal alignment. In this case, the wheel begins to oscillate about the steering axis at a frequency that is too high to be counteracted by the cyclist. The use of a simple linear relation between forces and angles, as stated in equations (1)-(6), is not sufficient. In fact, if the relation is linear, the oscillations are still present in the system response, but they are not limited in amplitude. As a consequence, both the rider and the bicycle fall in a few seconds. By adding instead a saturation at high angle values, the amplitude of the oscillations will remain limited in time.

Finally, some practical tips to be applied if the shimmy occurs are discussed. Overall, there is no way to stop a violent shimmy. These tips, however, are strongly recommended because they can contribute significantly to limit the amplitude of the oscillations. The first tip is the rider to assume an upright posture to increase the aerodynamic drag, thus promoting a deceleration of the bicycle. It is also suggested to tighten the horizontal tube of the rear frame with the legs, increasing in this way the structural stiffness. If necessary, gently use the rear brake. Usually, the oscillations are not divergent so it is difficult that they can lead to a fall, although this is the sensation perceived by the rider during the occurrence of the phenomenon.

\section{References}

Andreasson, J. (2003). "Vehicle Dynamics Library". In: Proceedings of the 3rd International Modelica Conference.

Åström, K. J., R. E. Klein, and A. Lennartsson (2005). "Bicycle dynamics and control". In: IEEE Control Systems Magazine 25.4, pp. 26-47.

Donida, F., G. Ferretti, S. M. Savaresi, F. Schiavo, and M. Tanelli (2006). "Motorcycle Dynamics Library in Modelica". In: Proceedings of 5th International Modelica Conference. Vienna, Austria, pp. 157-166.

Donida, F., G. Ferretti, S. M. Savaresi, and M. Tanelli (2008). "Object-oriented modelling and simulation of a motorcycle". In: Mathematical and Computer Modelling of Dynamical Systems 14.2, pp. 79-100.

Klinger, F., J. Nusime, J. Edelmann, and M. Plöchl (2014). "Wobble of a racing bicycle with a rider hands on and hands off the handlebar". In: Vehicle System Dynamics 52.

Limebeer, D. J. N. and R. S. Sharp (2006). "Bicycle, motorcycles, and models". In: IEEE Control Systems Magazine.

Magnani, G., N. M. Ceriani, and J. Papadopoulos (2013). "On-road measurements of high speed bicycle shimmy, and comparison to structural resonance". In: 2013 IEEE International Conference on Mechatronics, pp. 400405.

Pacejka, H. B. (1993). "The Magic Formula tire model". In: Vehicle System Dynamics (supplement) 21, pp. 118.

- (2006). Tire and Vehicle Dynamics. Ed. by Elsevier Ltd. second edition. Chap. chapter 4.

Plöchl, M., J. Edelmann, B. Angrosch, and C. Ott (2012). "On the wobble mode of a bicycle". In: Vehicle System Dynamics 50.3, pp. 415-429. 